\title{
microRNA expression profiling of heart tissue during fetal development
}

\author{
JIZI ZHOU $^{1 *}$, XINRAN DONG ${ }^{2 *}$, QIONGJIE ZHOU ${ }^{1}$, HUIJUN WANG $^{3,4}$, YANYAN QIAN ${ }^{3,4}$, \\ WEIDONG TIAN ${ }^{2}$, DUAN MA ${ }^{3,4}$ and XIAOTIAN LI ${ }^{1,4,5}$ \\ ${ }^{1}$ Obstetrics and Gynecology Hospital, ${ }^{2}$ Department of Biostatistics and Computational Biology, \\ School of Life Sciences, ${ }^{3}$ Key Laboratory of Molecular Medicine, Ministry of Education, Department of Biochemistry \\ and Molecular Biology, Shanghai Medical College, ${ }^{4}$ Institute of Biomedical Sciences, Fudan University; \\ ${ }^{5}$ Shanghai Key Laboratory of Female Reproductive Endocrine Related Diseases, Shanghai, P.R. China
}

Received October 26, 2013; Accepted February 18, 2014

DOI: $10.3892 /$ ijmm.2014.1691

\begin{abstract}
RNAs (miRNAs) are important both in early cardiogenesis and in the process of heart maturation. The aim of this study was to determine the stage-specific expression of miRNAs in human fetal heart in order to identify valuable targets for further study of heart defects. Affymetrix microarrays were used to obtain miRNA expression profiles from human fetal heart tissue at 5, 7, 9 and 23 weeks of gestation. To identify differentially expressed miRNAs at each timepoint, linear regression analysis by the $\mathrm{R}$ limma algorithm was employed. Hierarchical clustering analysis was conducted with Cluster 3.0 software. Gene Ontology analysis was carried out for miRNAs from different clusters. Commonalities in miRNA families and genomic localization were identified, and the differential expression of selected miRNAs from different
\end{abstract}

Correspondence to: Dr Xiaotian Li, Obstetrics and Gynecology Hospital, Fudan University, 419 Fangxie Road, Shanghai 200011, P.R. China

E-mail: xiaotianli555@163.com

Dr Duan Ma, Key Laboratory of Molecular Medicine, Ministry of Education, Department of Biochemistry and Molecular Biology, Shanghai Medical College, Fudan University, 138 Yixueyuan Road, Shanghai 200032, P.R. China

E-mail: duanma@fudan.edu.cn

Abbreviations: miRNAs, microRNAs; MEF2c, myocyte enhancer factor 2c; Tbx5, T-box 5; VEGFA, vascular endothelial growth factor $\alpha$; BMPR2, bone morphogenetic protein receptor 2; TGFBR2, transforming growth factor $\beta$ receptor 2; EGFR, epidermal growth factor receptor; HMGA2, high mobility group A2; Bcl-2, B cell lymphoma/ lewkmia-2; PDGF, platelet-derived growth factor; GO, Gene Ontology; RT-PCR, reverse transcription-polymerase chain reaction

*Contributed equally

Key words: microRNA expression, fetal heart development, gene ontology analysis, quantitative polymerase chain reaction clusters was verified by quantitative polymerase chain reaction (qPCR). A total of 703 miRNAs were expressed in human fetal heart. Of these, 288 differentially expressed miRNAs represented 5 clusters with different expression trends. Several clustered miRNAs also shared classification within miRNA families or proximal genomic localization. qPCR confirmed the expression patterns of selected miRNAs. miRNAs within the 5 clusters were predicted to target genes vital for heart development and to be involved in cellular signaling pathways that affect heart structure formation and heart-associated cellular events. In conclusion, to the best of our knowledge, this is the first miRNA expression profiling study of human fetal heart tissue. The stage-specific expression of specific miRNAs suggests potential roles at distinct time-points during fetal heart development.

\section{Introduction}

Heart development is a complicated spatio-temporal process of organ formation. The eventual anatomic formation of the heart crescent, linear heart tube, looped heart tube, and multichambered heart during the process of heart development depends on the coordination of regulatory mechanisms at the molecular level. Precise expression of heart genes is critical in specific events of cardiogenesis, and thus dysregulated gene expression can lead to a variety of heart defects (1). Although many studies have been conducted to investigate the genetic factors of heart development, the understanding of epigenetic mechanisms is currently limited.

microRNAs (miRNAs), as one of the epigenetic factors, have become acknowledged as new indirect regulators in heart development. miRNAs are endogenous $\sim 22$ nucleotide RNA species that target the mRNAs of protein-coding genes to direct repression activities at the post-translational level (2). Based on predictions of the target genes by bioinformatic analysis, it is estimated that miRNAs regulate at least $20 \%$ of human genes (3).

A number of studies have identified specific miRNAs in animal models that play distinct roles during heart development (4-7). Multiple miRNAs have been reported to play a 
vital role by regulating heart gene expression in heart development. For example, miR-1 and miR-133a, co-transcribed in heart cells, can occupy the Hand2 3'-UTR concurrently, regulating the expression of Hand2, an essential gene for heart development (8). In mouse models, miR-27b exhibits obvious myocardial expression during ventricular chamber formation by targeting the MEF2c gene. In zebrafish embryos, miR-218 is involved in the onset of heart malformation as a crucial mediator of Tbx5, a key gene mediating vertebrate heart development (9).

Notably, miRNAs have distinct expression patterns at different stages of development (10). miRNAs in zebrafish and rodent organs are reported to be expressed in a stage-specific manner $(11,12)$. There is evidence for a stage-specific role of miRNAs in heart development: mice with heart-specific deficiency of Dicer, a key miRNA-processing enzyme, have different abnormal heart phenotypes at different heart developmental stages $(13,14)$.

Therefore, stage-specific miRNA expression patterns are important for better predicting the roles for miRNAs in heart development. In this study, we aimed to establish the stagedependent expression patterns of miRNAs during human fetal heart development to provide valuable information for further investigations of congenital heart defects.

\section{Materials and methods}

Sample collection. Heart tissue from different weeks of gestation was obtained from aborted fetuses. The ages of the embryos and fetuses were carefully calculated after conception based on the last menstrual period, adjusting for ultrasound measurements of fetal biparietal diameter or crown rump length. Tissue at 5, 7 and 9 weeks of gestation (5W, 7W and 9W) was obtained from whole embryo hearts with the help of a dissecting microscope (Leica DFC290; Danaher Corp., Washington, DC, USA) and sets of 4 of the $5 \mathrm{~W}$ samples were pooled prior to processing because the amount of embryonic heart tissue at this early timepoint was minimal. The other time-points were processed as independent replicates. No obvious anatomical abnormalities were observed. Fetal heart tissue at 23 weeks of gestational age $(23 \mathrm{~W})$ was isolated at the conjunction site of the outflow tract, and ventricles and heart anatomy was confirmed normal by abdominal fetal echocardiography. To account for biological variability, a single pool of 4 samples at $5 \mathrm{~W}$, and 4,4 and 2 independent biological replicates of myocardium tissue at $7 \mathrm{~W}$, $9 \mathrm{~W}$ and $23 \mathrm{~W}$ were processed for microarray analysis. An additional pool of 4 samples, and 3, 3 and 3 replicates were used for qRT-PCR validation analysis. The study was approved by the Ethics Committee of the Obstetrics and Gynecology Hospital of Fudan University. All of the donors provided informed consent.

RNA extraction and quality control. Fetal myocardium tissue was incubated in RNA later solution (Qiagen, Valencia, CA, USA) at room temperature for $12 \mathrm{~h}$, and then stored at $-80^{\circ} \mathrm{C}$. RNA was extracted with TRIzol reagent (Invitrogen Life Technologies, Carlsbad, CA, USA) from 100 to $200 \mathrm{mg}$ of frozen tissue. An RNA quality control assessment was strictly performed prior to microarray and RT-PCR experiments as follows: RNA purity of $A 260 / 280 \geq 1.90$ was confirmed using a spectrophotometer (NanoDrop 1000; Thermo Scientific, Wilmington, MA, USA).
The integrity of total RNA was verified by agarose gel electrophoresis: rRNA $28 \mathrm{~S} / 18 \mathrm{~S}$ band brightness $\geq 2: 1$ or $1: 1$. The yield of total RNA for microarray experiments was verified to be $\geq 5 \mu \mathrm{g}$ for each sample as measured by spectrophotometry (Thermo Scientific NanoDrop 1000). A total of 2 pools of 5W and 7, 7 and 5 individual samples from $7 \mathrm{~W}, 9 \mathrm{~W}$ and $23 \mathrm{~W}$ passed this screen and were used in subsequent assays.

Microarray analysis. Microarray analysis was performed by CapitalBio Corp. (Beijing, China). After a brief tailing reaction with polyA, RNA samples were labeled by FlashTag ligation biotin mix. Labeled RNAs were hybridized overnight to Affymetrix GeneChip ${ }^{\circledR}$ miRNA 2.0 Arrays containing probes for 15,644 mature miRNAs derived from the Sanger miRBase V15 (from 131 organisms). Each array included probes for 1,105 human mature miRNAs. After hybridization, arrays were washed and stained according to standard Affymetrix protocol and then scanned on an Affymetrix GeneChip ${ }^{\circledR}$ Scanner 3000. Microarray data were preprocessed by extraction of the intensities for each individual miRNA followed by detection calls based on the Wilcoxon rank-sum test, background subtraction based on GC content of the anti-genomic probes, transformation of values through the addition of a small constant (value 16), quantile normalization and finally median summarization of all probe sets for each miRNA. The detection and background adjustments were conducted using the Affymetrix miRNA QC Tool, and the remaining workflow was performed under $\mathrm{R}$ programming environment (www.r-project.org). Reported intensity data were $\log 2$ transformed, and P-values were calculated by the two-sided Student's t-test. $\mathrm{P} \geq 0.06$ was considered to represent a higher than background probe signal, indicating the expression of the miRNA in fetal heart tissue.

Identification of differentially expressed miRNAs. miRNA expression levels in fetal heart tissue were compared for each of the six pairs of gestational ages $(5 \mathrm{~W}$ vs. $7 \mathrm{~W}, 5 \mathrm{~W}$ vs. $9 \mathrm{~W}, 5 \mathrm{~W}$ vs. $23 \mathrm{~W}, 7 \mathrm{~W}$ vs. $9 \mathrm{~W}, 7 \mathrm{~W}$ vs. $23 \mathrm{~W}$ and $5 \mathrm{~W}$ vs. $23 \mathrm{~W}$ ). Differentially expressed miRNAs were detected by limma, an R package based on linear regression. P-values were adjusted by the false discovery rate, and changes in miRNAs with $\mathrm{P}<0.05$ in any one of the comparisons was considered to indicate statistical significance. Expression profiles of 288 dynamically regulated miRNAs were determined by applied hierarchical clustering, and the miRNAs were grouped into 5 clusters with distinct patterns of expression during fetal heart morphogenesis. The above processes were accomplished using a self-designed $\mathrm{R}$ script.

Identification of miRNA families and miRNA genomics clustering. Enrichment analysis was performed using the Fisher's exact test to compare the identified miRNA clusters to the miRNA family dataset in miRFam (http://admis.fudan.edu.cn/ projects/miRFam.htm), which classifies 748 human miRNAs into 438 families. The four testing numbers were: total number of miRNAs annotated with a miRNA family; number of miRNAs in one of the miRNA clusters; number of miRNAs in a specific miRNA family; and number of miRNAs in the miRNA cluster also annotated within the specific miRNA family. Significance was set at $\mathrm{P}=0.01$. Since miRNAs located 
in close proximity to each other are highly co-expressed (15), we examined the genomic position for miRNAs. For each cluster, miRNAs located within $10 \mathrm{~kb}$ were treated as a single miRNA genomic cluster.

Target gene prediction and Gene Ontology (GO) enrichment. We predicted the target genes of the 288 miRNAs that were differentially expressed across four gestational ages with three online prediction tools: Target Scan (http://www.targetscan. org/), miRNAMap2 (http://www.targetscan.org/) and miRDB (http://mirnamap.mbc.nctu.edu.tw/). For each miRNA, target genes found in any one of the three online databases were considered for further analysis. GO enrichment analysis was performed for the target genes for each miRNA. We focused on biological process (BP), molecular function (MF) and cellular component (CC) branch GO terms that have 30-300 annotated genes. Significant P-values were obtained by Fisher's exact test in R, adjusted by the false discovery rate using a cut-off value of 0.001 .

Quantitative reverse transcription-polymerase chain reaction (qRT-PCR). To quantify the differential expression of gestation age-specific miRNAs, poly-A tails were added to total RNA samples from different gestation ages using E. coli polyA polymerase (NEB), as described previously (16). Then, $\sim 2 \mu \mathrm{g}$ of the tailed total RNA was reverse transcribed with ImProm-II (Promega, Madison, WI, USA). SYBR-Green (Takara, Shiga, Japan) qRT-PCR was performed using the Applied Biosystems 7900 real-time PCR system to assess miRNA expression with a specific forward primer and a universal reverse primer complementary to the anchor primer. The normalizer gene in this analysis was $18 \mathrm{~S}$ rRNA. The primers used are shown in Table I.

Statistical analysis. Normality of the data distribution was verified by the Kolmogorov-Smirnov test. Differences in the expression level of selected miRNAs in the fetal heart tissue from four gestational ages were validated using the t-test. Relative expression levels are expressed as the means \pm standard deviation (SD). Statistical significance was set at the $95 \%$ level $(\mathrm{P}<0.05)$.

\section{Results}

Differential miRNA expression profiling during fetal heart development. To identify miRNAs differentially expressed in the fetal heart during development, we performed expression profiling using Affymetrix Genechip ${ }^{\circledR}$ Arrays (Affymetrix Inc., Santa Clara, CA, USA) with 5, 7, 9 and 23 week-old fetal heart tissue. A total of 703 miRNAs were found to be expressed in developing fetal heart tissue. The 20 most highly expressed miRNAs over the four distinct gestational ages are listed in Table II. The expression of most miRNAs was not significantly altered throughout the fetal heart morphogenesis period; however, marked changes from 5 to 23 weeks of gestation age were observed in a subset of 288 miRNAs (Fig. 1A).

Hierarchical clustering analysis was performed to compare expression profiles of all miRNAs markedly regulated over the four time periods. Five distinguishable clusters were identifiable (Fig. 1B and C). Cluster 1 included 82 miRNAs that were
Table I. miRNA primer sequences used for qRT-PCR.

\begin{tabular}{ll} 
miRNA & \multicolumn{1}{c}{ Primer sequences } \\
\hline miRNA-20b & GGTAGCAAAGTGCTCATAGTGCAGGTAG \\
miRNA-504 & CTATCAGACCCTGGTCTGCACTCTATC \\
miRNA-302d & AGTGTTAAGTGCTTCCATGTTTGAGTGT \\
let-7a & TAGTTTGAGGTAGTAGGTTGTATAGTT \\
let-7b & TGGTTTGAGGTAGTAGGTTGTGTGGTT \\
let-7c & TGGTTTGAGGTAGTAGGTTGTATGGTT \\
let-7d & TAGTTAGAGGTAGTAGGTTGCATAGTT \\
18SpolyAF & AGTCGTAACAAGGTTTCCGTAGGTG
\end{tabular}

Universal reverse primer

miR-Hi-RE CCAGTCTCAGGGTCCGAGGTATTC

miRNA, microRNA; qRT-PCR, quantitative real-time-polymerase chain reaction.

highly expressed at 5 weeks of gestation, and then decreased with a fluctuating, uncharacteristic trend in the following three time-points. The 44 miRNAs in Cluster 2 exhibited a high expression across the first three time-points, followed by a low expression at 23 weeks of gestation. miRNAs in Clusters 3 and 4 contained 55 and 18 miRNAs with a high expression level at $7 \mathrm{~W}$ and $9 \mathrm{~W}$, respectively. The 89 miRNAs in Cluster 5 increased in expression, with the highest level at 23 weeks of gestational age. The miRNAs in the 5 different clusters are shown in Table III.

To assess the patterns of expression of miRNAs that have previously been reported to be associated with heart development, we identified relevant published studies by searching 'heart' and 'miRNA' on PubMed. Thirty-four of the miRNAs were associated with heart development (Table IV).

miRNA families and genomic clusters in 5 differentially expressed clusters. Co-expression of miRNAs is associated with sequence similarity and genomic co-localization (17). To determine whether patterns of expression correlate with genomic co-localization, we examined whether the miRNAs within expression clusters were localized within common miRNA families or genomic clusters. Five miRNA families with multiple differentially expressed miRNAs were identified (Table V), while many common genomic clusters were also observed (Table VI). These results support the possibility of the co-regulation of clustered miRNAs within miRNA families and genomic clusters.

Verification of miRNA expression patterns by qRT-PCR. To validate the microarray results, seven miRNAs predicted to be involved in heart development were selected for qRT-PCR based on their representation in two distinctive clusters and in a well-characterized miRNA family for the let-7 miRNAs. This validation was analyzed in 1 pool from $5 \mathrm{~W}$ and 3,3 and 3 individual samples from $7 \mathrm{~W}, 9 \mathrm{~W}$ and $23 \mathrm{~W}$ that we collected separately. miRNA-20b, miR-504 and miR-302d from Cluster 1 were expressed with a decreasing trend with gestational age (Fig. 2A). Conversely, the let-7 family miRNAs, let-7a, let-7b, let-7c and let-7d from Cluster 5 were expressed with a gradually 


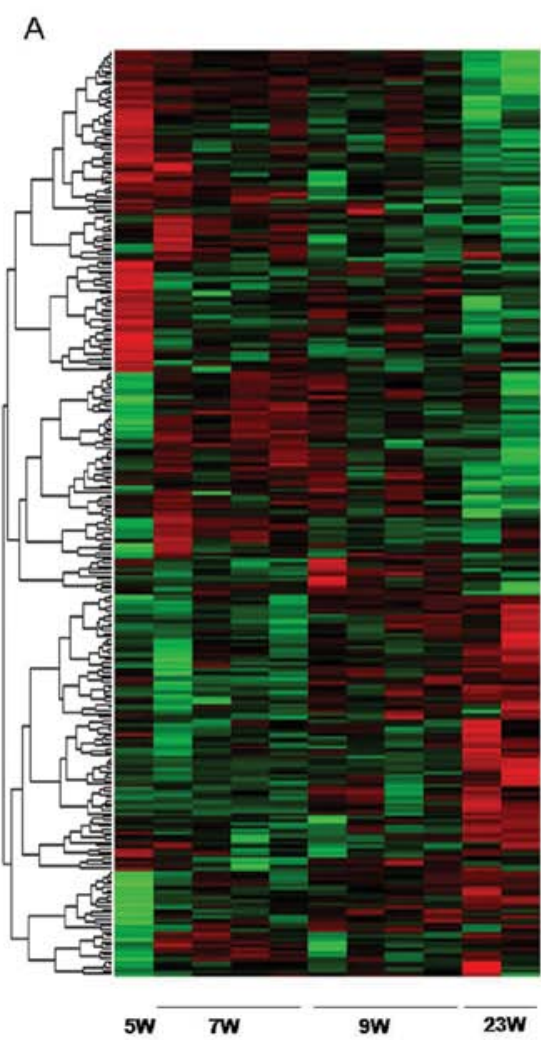

B

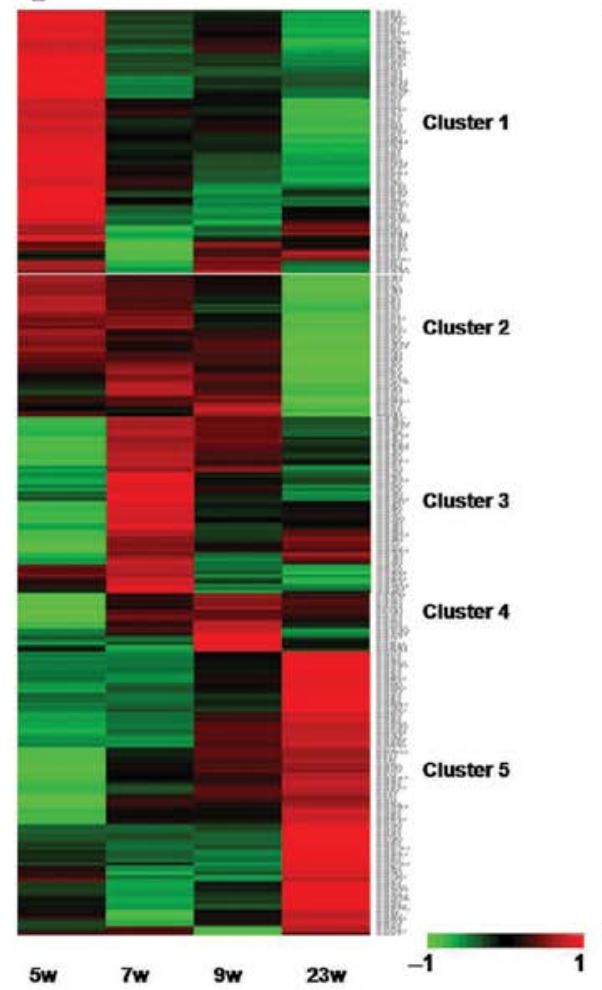

C

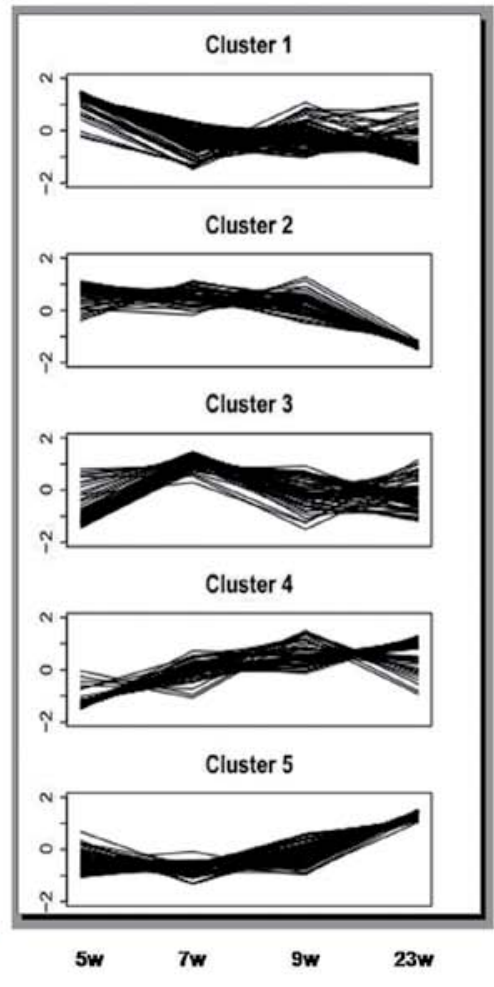

Figure 1. Microarray analysis of differentially expressed miRNAs in the developing fetal heart tissue. (A) Heatmap of the miRNAs that were found to be expressed differentially at the four time-points. Results for a single pool of 4 samples at 5 weeks (5W), and 4, 4 and 2 individual samples at 7, 9 and 23 weeks (7W, $9 \mathrm{~W}$ and $23 \mathrm{~W}$ ) are shown. Each column is a time-point, while each row represents an miRNA. Red to green indicates high to low expression. (B) Hierarchical clustering analysis of differentially expressed miRNAs from panel A revealed five prominent expression patterns throughout the gestational age. (C) Schematic diagram showing the changing trends of miRNAs within the 5 clusters.

increasing trend with gestational age (Fig. 2B). These trends are in agreement with the microarray results.

Function associations of miRNAs from 5 different expression clusters. To understand how differentially regulated miRNAs may contribute to fetal cardiogenesis and heart development, we analyzed the predicted functions of the miRNAs by enriching for predicted GO functions of target genes using online databases.

We focused on miRNAs with predicted roles in heart formation and development to obtain a complete network diagram (data not shown). The miRNAs within several clusters were predicted to target common genes (Fig. 3). This included the gene encoding vascular endothelial growth factor $\alpha$ (VEGFA) in Cluster 1, the bone morphogenetic protein receptor 2 (BMPR2) and transforming growth factor $\beta$ receptor 2 (TGFBR2) genes in Cluster 2, and epidermal growth factor receptor (EGFR) in Cluster 4. The miRNAs in Cluster 5 were predicted to target the high mobility group (HMGA2), Bcl-2 and VEGFA genes. These genes have associated roles in heart muscle tissue development, angiogenesis, outflow tract development, ventricle septum, heart chamber and ventricle morphogenesis. Common cellular events vital to cardiogenesis, such as the establishment and maintenance of cell polarity, cell response to growth factor, cell response to hypoxia, mesenchymal cell development and stem cell maintenance were also suggested by the GO annotation analysis. Furthermore, most targeted mRNAs were associated with cardiogenesis-related molecular signaling pathways, such as the Wnt, Notch, ERBB, PDGF, FGFR and retinoic acid receptor (RXR) signaling pathways (Fig. 3).

\section{Discussion}

We have characterized miRNA expression in the developing fetal heart over a period of 5-23 weeks of gestation. We identified 288 differentially expressed miRNAs, which clustered into 5 different expression patterns. Evidence was presented for the co-regulation of multiple miRNAs based on their categorization within miRNA families or localization within the genome. Based on GO, these miRNAs were predicted to target several common heart genes and to be associated with molecular events and signaling pathways during heart development.

To the best of our knowledge, this is the first study addressing miRNA expression profiling in human fetal heart tissue as early as 5 weeks of gestation. At 5 weeks of gestation the torsion and looping processing of the human heart is completed, the aortic sac is divided into two conducts, and the left ventricle begins to acquire its outflow tract (18). Therefore, miRNAs that are highly expressed at this relatively early timepoint (Clusters 1 and 2) may be essential for the anatomical orchestration of heart structures. The other selected developmental time-points cover a period of maturation that occurs after the formation of the major heart anatomical components. At a later stage in gestation, after the establishment of heart 
Table II. The top 20 miRNAs ranked by expression value in four distinct gestational ages.

\begin{tabular}{|c|c|c|}
\hline Gestation age & miRNA name & Signal value \\
\hline \multirow[t]{20}{*}{$5 \mathrm{~W}$} & hsa-miR-103 & 15006.54 \\
\hline & hsa-miR-26a & 13228.73 \\
\hline & hsa-miR-145 & 12726.34 \\
\hline & hsa-miR-17 & 12547.82 \\
\hline & hsa-miR-106a & 11815.90 \\
\hline & hsa-miR-24 & 11472.96 \\
\hline & hsa-miR-107 & 11252.73 \\
\hline & hsa-miR-23b & 10569.70 \\
\hline & hsa-miR-143 & 10455.16 \\
\hline & hsa-miR-92a & 10273.35 \\
\hline & hsa-miR-16 & 9893.96 \\
\hline & hsa-miR-20a & 9558.97 \\
\hline & hsa-miR-125b & 9121.01 \\
\hline & hsa-let-7e & 9103.76 \\
\hline & hsa-miR-23a & 8884.30 \\
\hline & hsa-miR-126 & 8827.87 \\
\hline & hsa-miR-99b & 8244.29 \\
\hline & hsa-miR-93 & 7993.56 \\
\hline & hsa-miR-181a & 7595.30 \\
\hline & hsa-miR-125a-5p & 7258.14 \\
\hline \multirow[t]{20}{*}{$7 \mathrm{~W}$} & hsa-miR-26a & 14296.97 \\
\hline & hsa-miR-103 & 13448.74 \\
\hline & hsa-miR-145 & 11919.23 \\
\hline & hsa-miR-143 & 11494.30 \\
\hline & hsa-miR-107 & 11381.93 \\
\hline & hsa-miR-24 & 10984.56 \\
\hline & hsa-let-7e & 10704.38 \\
\hline & hsa-miR-17 & 10662.18 \\
\hline & hsa-miR-23b & 10160.69 \\
\hline & hsa-miR-125b & 10079.82 \\
\hline & hsa-miR-106a & 9949.90 \\
\hline & hsa-let-7a & 9583.51 \\
\hline & hsa-miR-16 & 9484.89 \\
\hline & hsa-miR-126 & 8883.17 \\
\hline & hsa-let-7c & 8649.26 \\
\hline & hsa-miR-23a & 8523.80 \\
\hline & hsa-miR-20a & 8444.86 \\
\hline & hsa-miR-92a & 7580.87 \\
\hline & hsa-miR-1826 & 7473.76 \\
\hline & hsa-miR-3196 & 6661.93 \\
\hline \multirow[t]{11}{*}{$9 \mathrm{~W}$} & hsa-miR-26a & 14567.85 \\
\hline & hsa-miR-145 & 12769.74 \\
\hline & hsa-miR-24 & 12584.92 \\
\hline & hsa-miR-143 & 11991.22 \\
\hline & hsa-miR-23b & 11601.83 \\
\hline & hsa-let-7e & 11396.80 \\
\hline & hsa-miR-103 & 11348.86 \\
\hline & hsa-let-7a & 10556.03 \\
\hline & hsa-miR-17 & 9974.02 \\
\hline & hsa-miR-16 & 9871.69 \\
\hline & hsa-miR-23a & 9664.65 \\
\hline
\end{tabular}

Table II. Continued.

\begin{tabular}{|c|c|c|}
\hline Gestation age & miRNAs name & Signal value \\
\hline & hsa-let-7c & 9632.56 \\
\hline & hsa-miR-106a & 9414.99 \\
\hline & hsa-miR-107 & 9036.46 \\
\hline & hsa-miR-125b & 8924.02 \\
\hline & hsa-miR-126 & 8227.37 \\
\hline & hsa-let-7b & 8210.81 \\
\hline & hsa-let-7d & 8140.66 \\
\hline & hsa-miR-20a & 7818.96 \\
\hline & hsa-miR-92a & 7655.32 \\
\hline \multirow[t]{20}{*}{$23 \mathrm{~W}$} & hsa-miR-26a & 14905.89 \\
\hline & hsa-let-7a & 12766.60 \\
\hline & hsa-let-7b & 12451.81 \\
\hline & hsa-let-7c & 12401.25 \\
\hline & hsa-miR-23b & 12350.40 \\
\hline & hsa-miR-24 & 11917.27 \\
\hline & hsa-miR-145 & 11532.50 \\
\hline & hsa-miR-143 & 10717.41 \\
\hline & hsa-miR-16 & 10305.78 \\
\hline & hsa-let-7d & 10037.60 \\
\hline & hsa-miR-125b & 9849.52 \\
\hline & hsa-let-7e & 9538.46 \\
\hline & hsa-miR-103 & 9532.92 \\
\hline & hsa-miR-23a & 9348.89 \\
\hline & hsa-miR-126 & 8936.63 \\
\hline & hsa-miR-107 & 8528.02 \\
\hline & hsa-miR-1826 & 6534.73 \\
\hline & hsa-miR-17 & 6519.36 \\
\hline & hsa-miR-1975 & 6153.68 \\
\hline & hsa-miR-106a & 6004.38 \\
\hline
\end{tabular}

miRNA, microRNA; W, weeks

anatomy, the fetal heart continues to develop. For example, cardiomyocytes proliferate and enlarge to keep the heart growing (19), and atrioventricular and semilunar valves remodel into thin fibrous leaflets capable of enduring constantly changing haemodynamic forces (20). We suggest that miRNAs of Clusters 3, 4 and 5, which increase in expression and reach a peak later in gestation, may be associated with late heart development. Consistent with the age-dependent expression of miRNAs revealed in our study, the differential expression of miRNAs was observed in the hearts of young adult and old mice (21). However, we have provided additional information regarding the role of miRNAs in early heart development by assessing time-dependent expression alterations in the embryo. These collective findings suggest that different miRNAs may be required during key stages of fetal heart morphogenesis and development that extend beyond anatomical formation.

Within the 5 clusters, we have identified several miRNAs that were members of the same miRNA family or shared a chromosomal proximity. Several members of the let-7 family of conserved miRNAs were identified in Cluster 4 . The let-7 
Table III. miRNAs in each cluster.

Cluster no.

Cluster 1

Cluster 2

Cluster 3

Cluster 4

Cluster 5
miRNA name

hsa-miR-509-3-5p, hsa-miR-769-3p, hsa-miR-1226, hsa-miR-18a*, hsa-miR-93* , hsa-miR-149 hsa-miR-1307, hsa-miR-935, hsa-miR-181a-2*, hsa-miR-346, hsa-miR-514b-5p, hsa-miR-129-3p, hsa-miR-1180, hsa-miR-532-3p, hsa-miR-99b*, hsa-miR-509-3p, hsa-miR-425, hsa-miR-302d, hsa-miR-20b", hsa-miR-424*, hsa-miR-874, hsa-miR-92a-1 ${ }^{*}$, hsa-miR-339-5p, hsa-miR-127-3p, hsa-miR-501-5p, hsa-miR-431, hsa-miR-134, hsa-miR-2276, hsa-miR-500*, hsa-miR-99a*, hsa-miR-1270, hsa-miR-200c, hsa-miR-654-5p, hsa-miR-551a, hsa-miR-532-5p, hsa-miR-433, hsa-miR-99b, hsa-miR-500, hsa-miR-504, hsa-miR-1251, hsa-miR-3143, hsa-miR-1265, hsa-miR-342-3p, hsa-miR-409-5p, hsa-miR-103-as, hsa-miR-652, hsa-miR-421, hsa-miR-25*, hsa-miR-589*, hsa-miR-3139, hsa-miR-520c-5p, hsa-miR-330-3p, hsa-miR-766, hsa-miR-891a, hsa-miR-18b, hsa-miR-508-5p, hsa-miR-501-3p, hsa-miR-301b, hsa-miR-877, hsa-miR-1201, hsa-miR-432, hsa-miR-1301, hsa-miR-181a*, hsa-miR-484, hsa-miR-628-3p, hsa-miR-324-5p, hsa-miR-518f", hsa-miR-744, hsa-miR-758, hsa-miR-1296, hsa-miR-941, hsa-miR-20b, hsa-miR-193b", hsa-miR-485-5p, hsa-miR-574-3p, hsa-miR-216a, hsa-miR-340*, hsa-miR-30c-2*, hsa-miR-154, hsa-miR-3201, hsa-miR-379, hsa-miR-3200

hsa-miR-1910, hsa-miR-18a, hsa-miR-31, hsa-miR-708, hsa-miR-183, hsa-miR-182, hsa-miR-130b, hsa-miR-370, hsa-miR-1275, hsa-miR-3178, hsa-miR-887, hsa-miR-409-3p, hsa-miR-106b" , hsa-miR-106a, hsa-miR-383, hsa-miR-93, hsa-miR-181b, hsa-miR-20a, hsa-miR-125b-1 ${ }^{*}$, hsa-miR-638, hsa-miR-1915, hsa-miR-17, hsa-miR-2861, hsa-miR-4298, hsa-miR-106b, hsa-miR-1285, hsa-miR-205, hsa-miR-631, hsa-miR-671-5p, hsa-miR-100, hsa-miR-2277, hsa-miR-1469, hsa-miR-510, hsa-miR-374a, hsa-miR-4304, hsa-miR-103, hsa-miR-19a, hsa-miR-34c-3p, hsa-miR-32, hsa-miR-376b, hsa-miR-675, hsa-miR-125a-3p, hsa-miR-200b*, hsa-miR-155

hsa-miR-4299, hsa-miR-4286, hsa-miR-92b* , hsa-miR-1908, hsa-miR-1909, hsa-miR-1184, hsa-miR-1228*, hsa-miR-663, hsa-miR-572, hsa-miR-1274b, hsa-miR-3172, hsa-miR-3141, hsa-miR-720, hsa-miR-1268, hsa-miR-4281, hsa-miR-149*, hsa-miR-1225-5p, hsa-miR-3180-3p, hsa-miR-762, hsa-miR-3196, hsa-miR-1207-5p, hsa-miR-3126-5p, hsa-miR-1260b, hsa-miR-1973, hsa-miR-1280, hsa-miR-4284, hsa-miR-3197, hsa-miR-4269, hsa-miR-1308, hsa-miR-4324, hsa-miR-21, hsa-miR-921, hsa-miR-3162, hsa-miR-1246, hsa-miR-1972, hsa-miR-939, hsa-miR-218, hsa-miR-489, hsa-miR-374b, hsa-miR-150*, hsa-miR-513b, hsa-miR-4257, hsa-miR-488*, hsa-miR-886-5p, hsa-miR-1274a, hsa-miR-886-3p, hsa-miR-513a-5p, hsa-miR-3175, hsa-miR-1912, hsa-miR-107, hsa-miR-3195, hsa-miR-663b, hsa-miR-1260, hsa-miR-187", hsa-miR-4310 hsa-miR-1272, hsa-let-7d*, hsa-miR-3152, hsa-miR-548*, hsa-miR-1273, hsa-miR-499-5p, hsa-miR-3124, hsa-miR-320e, hsa-miR-1183, hsa-miR-548u, hsa-miR-885-3p, hsa-miR-548c-3p, hsa-miR-3128, hsa-miR-548a-5p, hsa-miR-363* , hsa-miR-7, hsa-miR-16-2*, hsa-miR-155*,

hsa-miR-215, hsa-miR-1, hsa-miR-26b, hsa-miR-297, hsa-miR-195*, hsa-let-7i, hsa-let-7a, hsa-miR-204, hsa-miR-10a*, hsa-let-7g, hsa-let-7f, hsa-miR-3154, hsa-let-7d, hsa-let-7b, hsa-miR-224, hsa-miR-139-5p, hsa-miR-98, hsa-miR-193a-5p hsa-miR-424, hsa-miR-30e, hsa-miR-422a, hsa-let-7c, hsa-miR-483-3p, hsa-miR-605 hsa-miR-452, hsa-miR-224*, hsa-miR-647, hsa-miR-150, hsa-miR-10a, hsa-miR-195, hsa-miR-497, hsa-miR-22*, hsa-miR-10b, hsa-miR-146a, hsa-miR-483-5p, hsa-miR-486-3p, hsa-miR-376c, hsa-miR-664*, hsa-miR-193a-3p, hsa-miR-371-5p, hsa-miR-22, hsa-miR-28-3p, hsa-miR-486-5p, hsa-miR-1827, hsa-miR-139-3p, hsa-miR-378c, hsa-miR-371-3p, hsa-miR-372, hsa-miR-373, hsa-miR-933, hsa-miR-29a, hsa-miR-3148, hsa-miR-381, hsa-miR-30d, hsa-miR-411, hsa-miR-338-5p, hsa-let-7i", hsa-miR-132*, hsa-miR-30b, hsa-miR-15a, hsa-miR-30a, hsa-miR-99a, hsa-miR-584, hsa-miR-125b-2*, hsa-miR-337-5p, hsa-miR-1277, hsa-miR-4306, hsa-miR-3169, hsa-miR-24-1 ${ }^{*}$, hsa-miR-363, hsa-miR-10b ${ }^{*}$, hsa-miR-192, hsa-miR-152, hsa-miR-760, hsa-miR-455-5p, hsa-miR-542-5p, hsa-miR-223, hsa-miR-20a*, hsa-miR-27b, hsa-miR-595, hsa-miR-451, hsa-miR-17*, hsa-miR-29b-2*, hsa-miR-299-5p, hsa-miR-1271, hsa-miR-2115*, hsa-miR-185, hsa-let-7f-1* , hsa-miR-625 
Table IV. The expression values of 34 miRNAs reported to be associated with heart in our microarray data.

\begin{tabular}{|c|c|c|c|c|c|}
\hline \multirow[b]{2}{*}{ miRNA name } & \multicolumn{4}{|c|}{$\begin{array}{l}\text { Expression value in our microarray data } \\
\text { Weeks of gestation }\end{array}$} & \multirow[b]{2}{*}{ Authors/(Refs.) } \\
\hline & $5 \mathrm{~W}$ & $7 \mathrm{~W}$ & $9 \mathrm{~W}$ & $23 \mathrm{~W}$ & \\
\hline miRNA-497 & 106.46 & 74.52 & 79.06 & 243.94 & Porrello (53) \\
\hline miRNA-195 & 472.86 & 469.39 & 482.04 & 1791.19 & Porrello (53) \\
\hline miRNA-15a & 706.54 & 642.36 & 729.14 & 1087.14 & Porrello (53) \\
\hline miRNA-15b & 3752.69 & 3619.41 & 3321.15 & 3095.05 & Porrello (53) \\
\hline miRNA-155 & 609.10 & 588.46 & 719.53 & 451.60 & Porrello (53) \\
\hline miRNA-17 & 12547.82 & 10662.18 & 9974.02 & 6519.36 & Porrello (53) \\
\hline miRNA-93 & 7993.56 & 6524.40 & 5446.11 & 3577.60 & Porrello (53) \\
\hline miRNA-208b & 261.65 & 208.86 & 243.61 & 166.51 & Porrello (53) \\
\hline miRNA-25 & 1699.03 & 1537.65 & 1264.74 & 1373.08 & Ventura et al (54) \\
\hline miRNA-363 & 468.42 & 427.85 & 411.53 & 717.82 & Ventura et al (54) \\
\hline let-7c & 5919.04 & 8649.26 & 9632.56 & 12401.25 & Vacchi-Suzzi et al (55) \\
\hline miRNA-125b & 9121.01 & 10079.82 & 8924.02 & 9849.52 & Vacchi-Suzzi et al (55) \\
\hline miRNA-744 & 720.20 & 514.15 & 483.37 & 274.01 & Vacchi-Suzzi et al (55) \\
\hline miRNA-328 & 42.56 & 36.72 & 42.25 & 41.47 & Vacchi-Suzzi et al (55) \\
\hline miRNA-199a-3p & 3252.41 & 5217.02 & 5019.48 & 5159.05 & Vacchi-Suzzi et al (55) \\
\hline miRNA-99b & 8244.29 & 5172.97 & 4812.78 & 3657.86 & Vacchi-Suzzi et al (55) \\
\hline miRNA-30e & 322.33 & 344.42 & 501.56 & 712.99 & Vacchi-Suzzi et al (55) \\
\hline miRNA-30e & 112.36 & 146.29 & 161.25 & 180.60 & Vacchi-Suzzi et al (55) \\
\hline miRNA-21 & 217.27 & 393.67 & 321.76 & 337.54 & Huang et al (56) \\
\hline miRNA-22 & 2223.63 & 1629.95 & 2363.51 & 3026.23 & Tu et al (57) \\
\hline miRNA-126 & 8827.87 & 8883.17 & 8227.37 & 8936.63 & Stankunas et al (58) \\
\hline miRNA-452 & 36.70 & 53.57 & 69.68 & 118.69 & Sheehy et al (59) \\
\hline miRNA-378 & 2174.08 & 2255.47 & 3417.05 & 4984.94 & Nagalingam et al (60) \\
\hline miRNA-138 & 51.16 & 43.98 & 53.24 & 72.48 & Morton et al (6) \\
\hline miRNA-34a & 213.74 & 164.51 & 170.12 & 191.67 & Boon et al (61) \\
\hline miRNA-181c & 154.76 & 151.84 & 165.73 & 167.21 & Li et al (62) \\
\hline miRNA-204 & 48.61 & 87.33 & 86.48 & 182.21 & Xiao et al (63) \\
\hline miRNA-133b & 1778.10 & 1685.77 & 1891.50 & 2353.18 & Townley-Tilson et al (64) \\
\hline miRNA-133a & 3793.49 & 3548.94 & 3533.02 & 4250.38 & Townley-Tilson et al (64) \\
\hline miRNA-206 & 149.01 & 178.31 & 207.56 & 171.54 & Townley-Tilson et al (64) \\
\hline miRNA-1 & 665.07 & 1591.58 & 1655.17 & 1996.21 & Townley-Tilson et al (64) \\
\hline miRNA-143 & 10455.16 & 11494.30 & 11991.22 & 10717.41 & Deacon et al (4) \\
\hline miRNA-218 & 71.71 & 129.09 & 90.52 & 86.99 & Chiavacci et al (9) \\
\hline miRNA-208a & 36.03 & 32.93 & 37.72 & 44.18 & Oliveira-Carvalho et al (65) \\
\hline
\end{tabular}

miRNA, microRNA.

Table V. miRNA families within expression clusters.

\begin{tabular}{lllr}
\hline Cluster ID & miRNA family & \multicolumn{1}{c}{ Members of miRNA family } & P-value \\
\hline Cluster 2 & mir-17 & hsa-miR-20a, hsa-miR-18a, hsa-miR-93, hsa-miR-106a, hsa-miR-106b, hsa-miR-17 & 6.59E-08 \\
Cluster 3 & mir-1274 & hsa-miR-1274a, hsa-miR-1274b & $9.06 \mathrm{E}-04$ \\
& mir-663 & hsa-miR-663, hsa-miR-663b & $9.06 \mathrm{E}-04$ \\
Cluster 4 & let-7 & hsa-miR-98, hsa-let-7g, hsa-let-7b, hsa-let-7d, hsa-let-7c, hsa-let-7i & $1.89 \mathrm{E}-07$ \\
Cluster 5 & mir-30 & hsa-miR-30a, hsa-miR-30b, hsa-miR-30d, hsa-miR-30e & $4.45 E-05$
\end{tabular}

miRNA, microRNA. 
Table VI. miRNA genomic-clusters in each expression cluster.

\begin{tabular}{|c|c|c|c|c|}
\hline Cluster ID & Chromosome & Position at $5^{\prime}$ & Position at $3^{\prime}$ & Members of genomic-clusters \\
\hline \multirow[t]{9}{*}{ Cluster 1} & chr14 & 101350820 & 101350913 & hsa-miR-432, hsa-miR-433, hsa-miR-127-3p, hsa-miR-431 \\
\hline & $\operatorname{chr} 14$ & 101492357 & 101492444 & hsa-miR-758, hsa-miR-379 \\
\hline & chr14 & 101526092 & 101526175 & hsa-miR-485-5p, hsa-miR-134, hsa-miR-154 \\
\hline & chr19 & 52195865 & 52195934 & hsa-miR-99b*, hsa-miR-99b \\
\hline & chr19 & 54210707 & 54210793 & hsa-miR-518f*, hsa-miR-520c-5p \\
\hline & $\operatorname{chr} 7$ & 99691391 & 99691470 & hsa-miR-93* , hsa-miR-25* \\
\hline & $\operatorname{chrX}$ & 49774330 & 49774413 & $\begin{array}{l}\text { hsa-miR-501-3p, hsa-miR-532-5p, hsa-miR-500*, } \\
\text { hsa-miR-532-3p, hsa-miR-501-5p, hsa-miR-500 }\end{array}$ \\
\hline & $\operatorname{chrX}$ & 133304071 & 133304141 & hsa-miR-20b*, hsa-miR-20b, hsa-miR-18b \\
\hline & $\operatorname{chrX}$ & 146341170 & 146341244 & hsa-miR-514b-5p, hsa-miR-509-3-5p \\
\hline \multirow[t]{3}{*}{ Cluster 2} & $\operatorname{chr} 13$ & 92003319 & 92003389 & hsa-miR-17, hsa-miR-20a, hsa-miR-18a, hsa-miR-19a \\
\hline & $\operatorname{chr} 7$ & 99691616 & 99691697 & hsa-miR-93, hsa-miR-106b* , hsa-miR-106b \\
\hline & $\operatorname{chr} 7$ & 129414745 & 129414854 & hsa-miR-182, hsa-miR-183 \\
\hline Cluster 3 & $\operatorname{chr} 5$ & 135416177 & 135416297 & hsa-miR-886-5p, hsa-miR-886-3p \\
\hline \multirow[t]{2}{*}{ Cluster 4} & $\operatorname{chr} 17$ & 46657200 & 46657309 & hsa-miR-10a*, hsa-miR-10a \\
\hline & chr9 & 96941116 & 96941202 & hsa-let-7d, hsa-let-7d* \\
\hline \multirow[t]{11}{*}{ Cluster 5} & chr11 & 72326107 & 72326174 & hsa-miR-139-5p, hsa-miR-139-3p \\
\hline & $\operatorname{chr} 13$ & 92003319 & 92003389 & hsa-miR-20a*, hsa-miR-17* \\
\hline & $\operatorname{chr} 14$ & 101490131 & 101490193 & hsa-miR-411, hsa-miR-299-5p \\
\hline & $\operatorname{chr} 14$ & 101512257 & 101512331 & hsa-miR-381, hsa-miR-376c \\
\hline & $\operatorname{chr} 17$ & 1617197 & 1617281 & hsa-miR-22, hsa-miR-22* \\
\hline & $\operatorname{chr} 17$ & 6921230 & 6921341 & hsa-miR-497, hsa-miR-195 \\
\hline & chr19 & 54291959 & 54292027 & hsa-miR-372, hsa-miR-371-3p, hsa-miR-373, hsa-miR-371-5p \\
\hline & $\operatorname{chr} 2$ & 177015031 & 177015140 & hsa-miR-10b, hsa-miR-10b* \\
\hline & chr8 & 41517959 & 41518026 & hsa-miR-486-3p, hsa-miR-486-5p \\
\hline & chr8 & 135817119 & 135817188 & hsa-miR-30d, hsa-miR-30b \\
\hline & $\operatorname{chrX}$ & 151128100 & 151128184 & hsa-miR-224*, hsa-miR-452 \\
\hline
\end{tabular}

miRNA, microRNA.

family has diverse biological activities. We confirmed the high expression of let-7a, let-7b, let-7c and let-7d in human fetal heart tissue by qRT-PCR. We also confirmed the expression patterns of miRNA-20b, miRNA-504 and miRNA-302d. The functional analysis of these miRNAs may help to specify their roles in fetal heart development.

Results of our study suggest a role for miRNAs in the morphogenesis of the heart chamber, ventricle septum and outflow tract, as supported by previous studies. miRNA-143 is known to be essential for chamber formation and function through the active adjustment of myocardial cell morphology in zebrafish lines (4). Furthermore, miRNA-1 can indirectly control the balance between muscle differentiation and proliferation during cardiogenesis (22). Deletion of miRNA-133 in mice results in late embryonic or neonatal lethality due to ventricle septum defects, accompanied by abnormalities in cardiomyocyte proliferation, apoptosis and the aberrant expression of smooth muscle genes in the heart (5). In the present study, we have identified new miRNAs that are likely to be involved in the heart chamber, septum and outflow tract development through regulating the biological behavior of the muscle system.
Cell polarity is a feature in early organ patterning of the embryo $(23,24)$. It regulates the polarization of cells in a variety of contexts, allowing cells to change shape and position and to sense their orientation within a mass of tissue (25). The disruption of cell polarity is a known mechanism of heart defect (26). Several miRNAs identified in Clusters 1, 2, 3 and 4 may function in the establishment and maintenance of cell polarity. In addition, some miRNAs in Clusters 2 and 5 are regulated in response to hypoxia or oxygen levels. Thus, when the fetal heart is formed, it undergoes a stage of rapid growth and maturation where oxygen tension plays a vital role, and physiological normal hypoxia (lower oxygen tension in the fetus as compared with the adult) may be helpful in heart development (27).

The present study has identified genes previously involved in heart development as predicted targets of differentially expressed miRNAs. For example, VEGF-A, BMPR2,TGFBR2, EGFR, HMGA2, Bcl-2 were identified as target genes for multiple miRNAs within specific clusters. VEGF is involved in coronary vasculature, septation and outflow tract formation and influences cardiomyocyte survival (28). VEGF expression, 

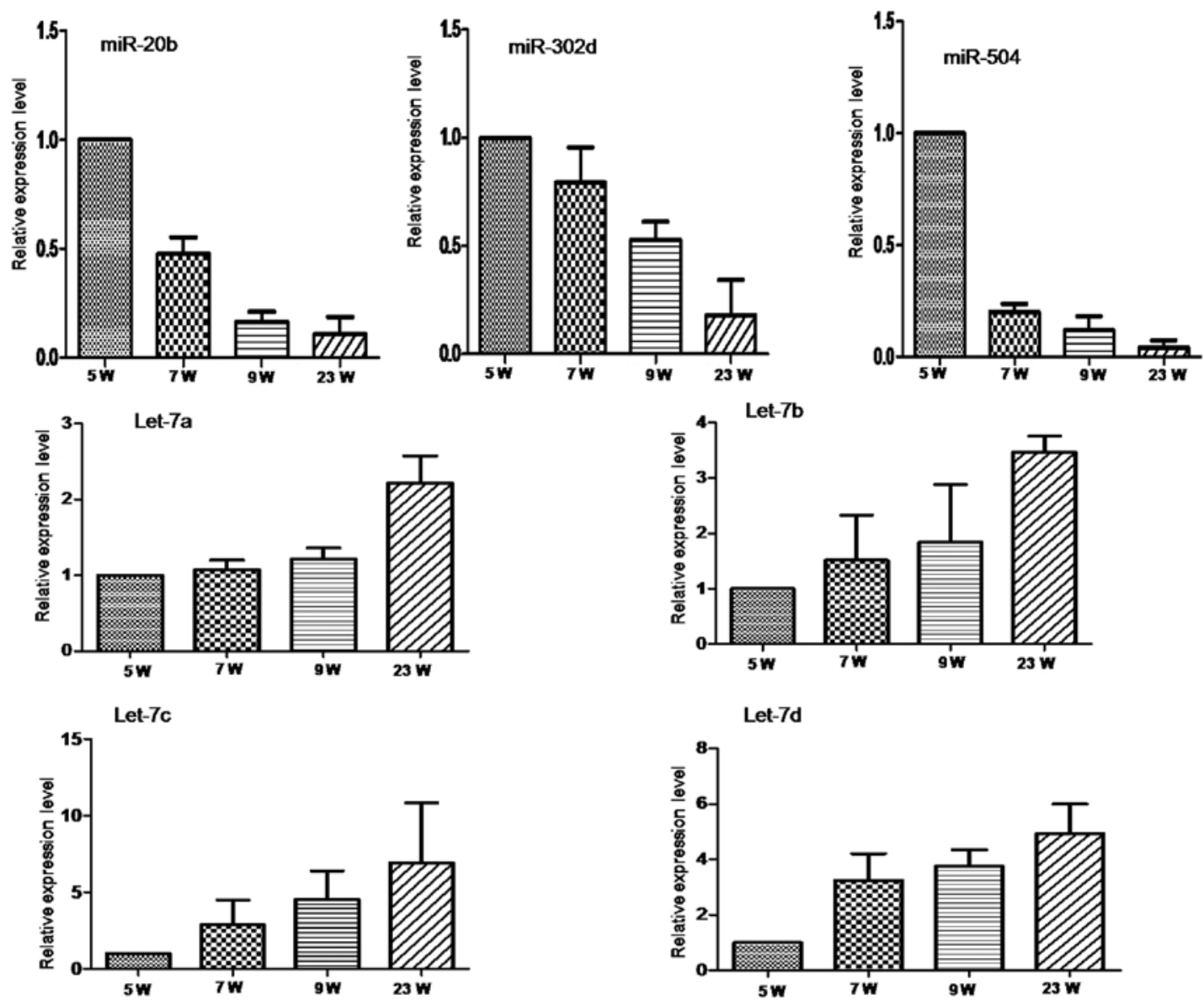

Figure 2. Verification of the expression patterns of 3 miRNAs from Cluster 1 and 4 miRNAs from Cluster 5 by qRT-PCR. qRT-PCR was performed using an additional set of samples that was independent from the samples used for microarray. Relative expression values of the 7 miRNAs were standardized to $18 \mathrm{~S}$ rRNA expression and normalized to 1 in the $5 \mathrm{~W}$ sample. Results present the means \pm SD of 3 replicates of the $7 \mathrm{~W}, 9 \mathrm{~W}$ and $23 \mathrm{~W}$ samples. W, weeks

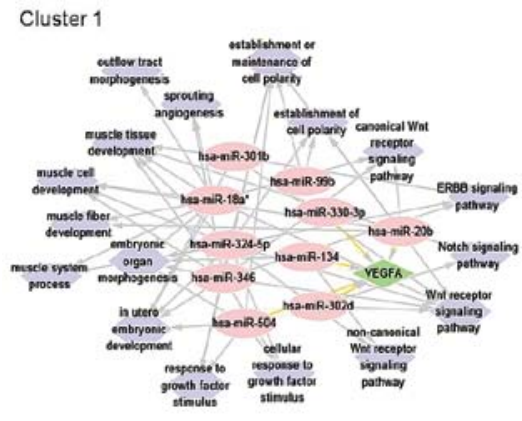

Cluster 3

Cluster 2
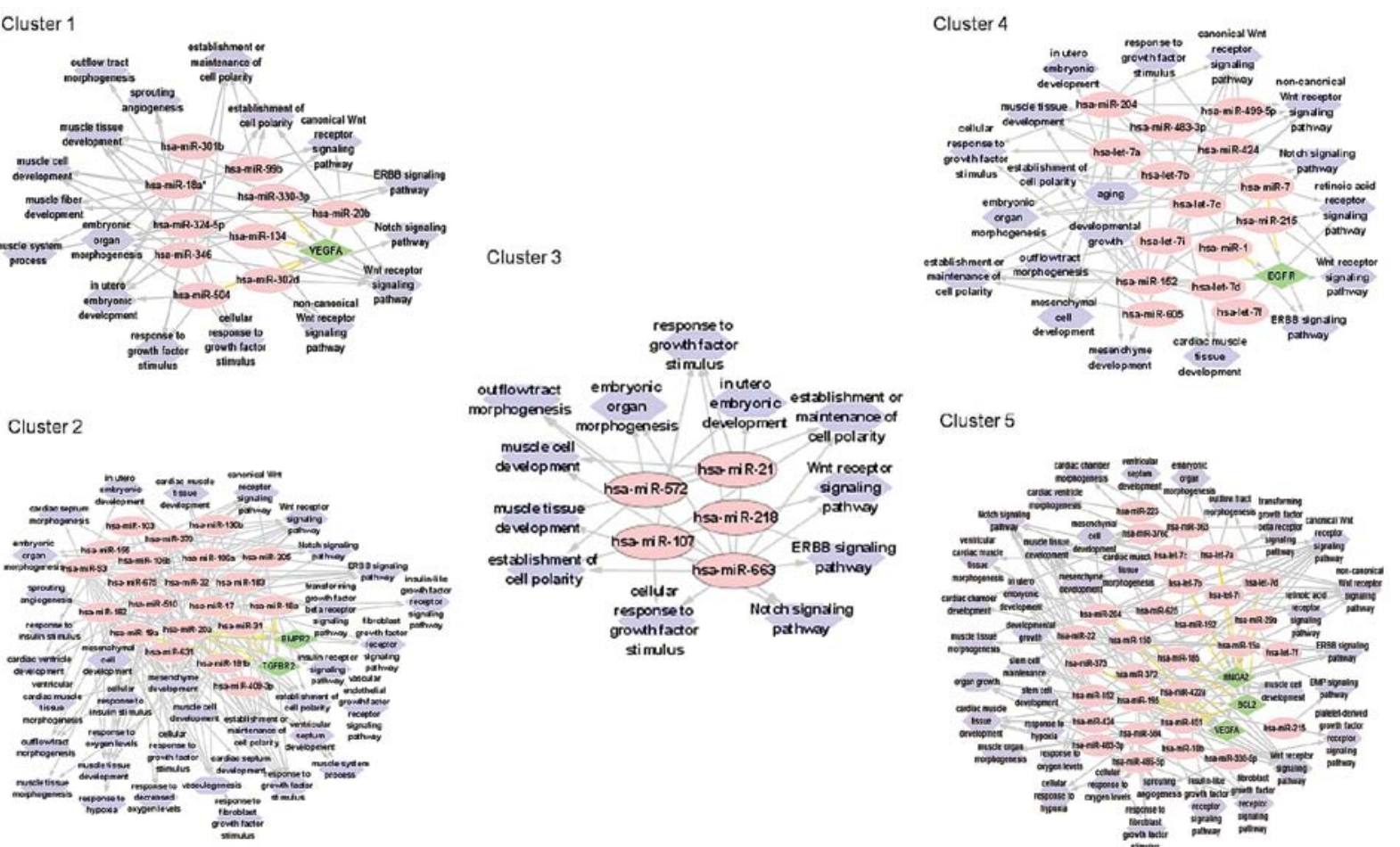

Figure 3. Interaction network diagrams of miRNAs in 5 clusters. miRNAs are shown by the pink ellipse boxes. Their predicted functions in biological processes and cellular activities associated with heart development correspond to the left-most light blue boxes and reported signaling pathways to the right-most light blue boxes. Green boxes are the predicted target genes. 
at either the mRNA or protein level, has been observed in rat hearts from the first embryonic day of myocardial vascular tube formation through the entire pregnancy (29). Therefore, miRNAs from Clusters 1 and 5 may regulate heart development by targeting VEGF. In animal models, inactivation of BMPR2, TGFBR2 and EGFR causes different subtypes of heart defects (30-32). Furthermore, HMGA2, a member of the HMGA sub-family of HMG proteins has a critical function for normal heart development (33). Accumulating evidence has revealed focal apoptosis in multiple cells of developing heart, contributing to normal development of embryonic outflow tract, heart valves, heart vascular system, and the conducting system (34). Bcl-2 is a common mediator of apoptosis that resides within the mitochondria and regulates cytochrome c release and caspase activation in the intrinsic apoptotic pathway (35). Several miRNAs, including let-7a, miRNA-204, miRNA-15a and miRNA-195 regulate the expression of Bcl-2 to influence apoptosis in certain diseases (36-39). Thus, the expression of these miRNAs in the fetal heart may have a similar function in influencing apoptosis.

Heart formation and development is known to be a complex process including numerous signaling pathways and their interactions. Through network and GO analysis, the differentially regulated miRNAs are shown to have a putative role in the regulation of heart development-associated signaling pathways, such as the canonical and non-canonical Wnt signaling pathway (40), ERBB (41), Notch $(28,42)$, TGF- $\beta$ (43), retinoic acid receptor (44), BMP (43,45), PDGF (46), FGF (47) and insulin-like growth factor receptor signaling pathways (48). Previous studies have reported a connection between miRNAs and many of these signaling pathways. For instance, miRNA499 induces rat bone marrow-derived mesenchymal stem cell differentiation in cardiomyocyte-like cells through the Wnt/ $\beta$-catenin signaling pathway (49). Extensive cross talk between miRNAs and the Notch signaling pathway determines stem cell fates (50). In addition, several miRNAs are shown to regulate molecular members of the ERBB signaling pathway in various types of cancer (51). The Wnt, ERBB and TGF- $\beta$ signaling pathways have also been predicted to be regulated by miR-335 in gastric cancer (52). Our results provide insight into additional miRNAs that may regulate heart development by these predicted signaling pathways.

In summary, we have identified a set of miRNAs that are expressed in a time-specific manner during the fetal period in the human developing heart. Using clustering and GO analyses, we have predicted the functions of differentially expressed miRNAs. These data elucidate the potential role of a network of miRNAs in anatomical and post-anatomical heart development and may provide insight into potential treatments of heart defects.

\section{Acknowledgements}

This study was supported by the National Basic Research Program of China (973 program) (2010CB529500) and the National Science Fund of China (81270712, 81300506, 81200449 and 81200448$)$. We gratefully acknowledge the assistance provided by the National Academic-Specific Program of Health Care, the Public Health Care Program of Shanghai (12GWZX0301) from Shanghai Municipal Health
Bureau, and the training program of the Shanghai Academic Leading Talent by the Shanghai Committee of Science and Technology and Shanghai Bureau of Human Resources.

\section{References}

1. Huang JB, Liu YL, Sun PW, Lv XD, Du M and Fan XM: Molecular mechanisms of congenital heart disease. Cardiovasc Pathol 19: e183-e193,2010.

2. Bartel DP: microRNAs: target recognition and regulatory functions. Cell 136: 215-233, 2009.

3. Xie X, Lu J, Kulbokas E, et al: Systematic discovery of regulatory motifs in human promoters and 3' UTRs by comparison of several mammals. Nature 434: 338-345, 2005.

4. Deacon DC, Nevis KR, Cashman TJ, etal: The miR-143-adducin3 pathway is essential for cardiac chamber morphogenesis. Development 137: 1887-1896, 2010.

5. Liu N, Bezprozvannaya S, Williams AH, et al: microRNA-133a regulates cardiomyocyte proliferation and suppresses smooth muscle gene expression in the heart. Genes Dev 22: 3242-3254, 2008.

6. Morton SU, Scherz PJ, Cordes KR, Ivey KN, Stainier DY and Srivastava D: microRNA-138 modulates heart patterning during embryonic development. Proc Natl Acad Sci USA 105: 1783017835,2008

7. Lagendijk AK, Goumans MJ, Burkhard SB and Bakkers J: MicroRNA-23 restricts heart valve formation by inhibiting Has2 and extracellular hyaluronic acid production. Circ Res 109: 649-657, 2011.

8. Vo NK, Dalton RP, Liu N, Olson EN and Goodman RH: Affinity purification of microRNA-133a with the heart transcription factor, Hand2. Proc Natl Acad Sci USA 107: 19231-19236, 2010.

9. Chiavacci E, Dolfi L, Verduci L, et al: MicroRNA 218 mediates the effects of Tbx 5a over-expression on zebrafish heart development. PLoS One 7: e50536, 2012.

10. Bartel DP: microRNAs: genomics, biogenesis, mechanism, and function. Cell 116: 281-297, 2004.

11. Wienholds E, Kloosterman WP, Miska E, et al: MicroRNA expression in zebrafish embryonic development. Science 309: 310-311, 2005.

12. Mukhopadhyay P, Brock G, Pihur V, Webb C, Pisano MM and Greene RM: Developmental microRNA expression profiling of murine embryonic orofacial tissue. Birth Defects Res A Clin Mol Teratol 88: 511-534, 2010.

13. Chen JF, Murchison EP, Tang R, et al: Targeted deletion of Dicer in the heart leads to dilated cardiomyopathy and heart failure. Proc Natl Acad Sci USA 105: 2111-2116, 2008.

14. Saxena A and Tabin CJ: miRNA-processing enzyme Dicer is necessary for heart outflow tract alignment and chamber septation. Proc Natl Acad Sci USA 107: 87-91, 2010.

15. Marco A, Ninova M, Ronshaugen M and Griffiths-Jones S: Clusters of microRNAs emerge by new hairpins in existing transcripts. Nucleic Acids Res 41: 7745-7752, 2013.

16. Zhang J, Du YY, Lin YF, et al: The cell growth suppressor, mir-126, targets IRS-1. Biochem Biophys Res Commun 377: 136-140, 2008.

17. Stäehler CF, Keller A, Leidinger P, et al: Whole miRNome-wide differential co-expression of microRNAs. Genomics Proteomics Bioinformatics 10: 285-294, 2012.

18. Marcela SG, Cristina RM, Angel PG, et al: Chronological and morphological study of heart development in the rat. Anat Rec (Hoboken) 295: 1267-1290, 2012.

19. Jonker SS, Zhang L, Louey S, Giraud GD, Thornburg KL and Faber JJ: Myocyte enlargement, differentiation, and proliferation kinetics in the fetal sheep heart. J Appl Physiol 102: 1130-1142, 2007.

20. Butcher JT and Markwald RR: Valvulogenesis: the moving target. Philos Trans R Soc Lond B Biol Sci 362: 1489-1503, 2007.

21. Zhang X, Azhar G and Wei JY: The expression of microRNA and microRNA clusters in the aging heart. PLoS One 7: e34688, 2012.

22. Zhao Y, Samal E and Srivastava D: Serum response factor regulates a muscle-specific microRNA that targets Hand 2 during cardiogenesis. Nature 436: 214-220, 2005.

23. Sinha T, Wang B, Evans S, Wynshaw-Boris A and Wang J: Disheveled mediated planar cell polarity signaling is required in the second heart field lineage for outflow tract morphogenesis. Dev Biol 370: 135-144, 2012. 
24. Zagris N, Gilipathi K, Soulintzi N and Konstantopoulos K: Decorin developmental expression and function in the early avian embryo. Int J Dev Biol 55: 633-639, 2011.

25. Henderson DJ and Chaudhry B: Getting to the heart of planar cell polarity signaling. Birth Defects Res A Clin Mol Teratol 91: 460-467, 2011

26. Rhee DY, Zhao XQ, Francis RJ, Huang GY, Mably JD and Lo CW: Connexin 43 regulates epicardial cell polarity and migration in coronary vascular development. Development 136 3185-3193, 2009

27. Patterson AJ and Zhang L: Hypoxia and fetal heart development. Curr Mol Med 10: 653-666, 2010

28. van den Akker NM, Caolo V and Molin DG: Cellular decisions in heart outflow tract and coronary development: an act by VEGF and NOTCH. Differentiation 84: 62-78, 2012

29. Tomanek RJ, Ratajska A, Kitten GT, Yue X and Sandra A: Vascular endothelial growth factor expression coincides with coronary vasculogenesis and angiogenesis. Dev Dyn 215: 54-61, 1999.

30. Beppu H, Malhotra R, Beppu Y, Lepore JJ, Parmacek MS and Bloch KD: BMP type II receptor regulates positioning of outflow tract and remodeling of atrioventricular cushion during cardiogenesis. Dev Biol 331: 167-175, 2009.

31. Langlois D, Hneino M, Bouazza L, et al: Conditional inactivation of TGF- $\beta$ type II receptor in smooth muscle cells and epicardium causes lethal aortic and heart defects. Transgenic Res 19: 1069-1082, 2010

32. Goishi K, Lee P, Davidson AJ, Nishi E, Zon LI and Klagsbrun M: Inhibition of zebrafish epidermal growth factor receptor activity results in cardiovascular defects. Mech Dev 120: 811-822, 2003.

33. Monzen K, Ito Y, Naito AT, et al: A crucial role of a high mobility group protein HMGA2 in cardiogenesis. Nat Cell Biol 10: $567-574,2008$

34. Fisher SA, Langille BL and Srivastava D: Apoptosis during cardiovascular development. Circ Res 87: 856-864, 2000.

35. Vogler M: BCL2A1: the underdog in the BCL2 family. Cell Death Differ 19: 67-74, 2012.

36. Vasilatou D, Papageorgiou SG, Kontsioti F, et al: Expression analysis of mir-17-5p, mir-20a and let-7a microRNAs and their target proteins in $\mathrm{CD} 34^{+}$bone marrow cells of patients with myelodysplastic syndromes. Leuk Res 37: 251-258, 2013.

37. Ryan J, Tivnan A, Fay J, et al: MicroRNA-204 increases sensitivity of neuroblastoma cells to cisplatin and is associated with a favourable clinical outcome. Br J Cancer 107: 967-976, 2012.

38. Diniz MG, Gomes CC, de Castro WH, et al: miR-15a/16-1 influences BCL2 expression in keratocystic odontogenic tumors. Cell Oncol (Dordr) 35: 285-291, 2012.

39. Chen YQ, Wang XX, Yao XM, et al: MicroRNA-195 promotes apoptosis in mouse podocytes via enhanced caspase activity driven by BCL2 insufficiency. Am J Nephrol 34: 549-559, 2011.

40. Cohen ED, Tian Y and Morrisey EE: Wnt signaling: an essentia regulator of cardiovascular differentiation, morphogenesis and progenitor self-renewal. Development 135: 789-798, 2008.

41. Odiete O, Hill MF and Sawyer DB: Neuregulin in cardiovascular development and disease. Circ Res 111: 1376-1385, 2012.

42. Jain R, Rentschler S and Epstein JA: Notch and heart outflow tract development. Ann NY Acad Sci 1188: 184-190, 2010.

43. Kruithof BP, Duim SN, Moerkamp AT and Goumans MJ: TGF and BMP signaling in heart cushion formation: lessons from mice and chicken. Differentiation 84: 89-102, 2012.

44. Li P, Pashmforoush M and Sucov HM: Retinoic acid regulates differentiation of the secondary heart field and TGFbetamediated outflow tract septation. Dev Cell 18: 480-485, 2010.

45. Zhang J, Chang JY, Huang Y, et al: The FGF-BMP signaling axis regulates outflow tract valve primordium formation by promoting cushion neural crest cell differentiation. Circ Res 107: $1209-1219,2010$
46. Van den Akker NM, Winkel LC, Nisancioglu MH, et al: PDGF-B signaling is important for murine heart development: its role in developing atrioventricular valves, coronaries, and heart innervation. Dev Dyn 237: 494-503, 2008

47. Lavine KJ, Yu K, White AC, et al: Endocardial and epicardial derived FGF signals regulate myocardial proliferation and differentiation in vivo. Dev Cell 8: 85-95, 2005.

48. Knezevic I, Patel A, Sundaresan NR, et al: A novel cardiomyocyteenriched microRNA, miR-378, targets insulin-like growth factor 1 receptor: implications in postnatal heart remodeling and cell survival. J Biol Chem 287: 12913-12926, 2012.

49. Zhang LL, Liu JJ, Liu F, et al: MiR-499 induces heart differentiation of rat mesenchymal stem cells through wnt/beta-catenin signaling pathway. Biochem Biophys Res Commun 420: 875-881, 2012.

50. Ichimura A, Ruike Y, Terasawa K and Tsujimoto G: miRNAs and regulation of cell signaling. FEBS J 278: 1610-1618, 2011.

51. Barker A, Giles KM, Epis MR, Zhang PM, Kalinowski F and Leedman PJ: Regulation of ErbB receptor signalling in cancer cells by microRNA. Curr Opin Pharmacol 10: 655-661, 2010.

52. Yan Z, Xiong Y, Xu W, et al: Identification of hsa-miR-335 as a prognostic signature in gastric cancer. PLoS One 7: e40037, 2012.

53. Porrello ER: microRNAs in heart development and regeneration. Clin Sci (Lond) 125: 151-166, 2013.

54. Ventura A, Young AG, Winslow MM, et al: Targeted deletion reveals essential and overlapping functions of the miR-17 through 92 family of miRNA clusters. Cell 132: 875-886, 2008.

55. Vacchi-Suzzi C,HahneF,ScheubelP,etal:Heartstructure-specific transcriptomic atlas reveals conserved microRNA-mRNA interactions. PloS One 8: e52442, 2013.

56. Huang ZP, Chen JF, Regan JN, et al: Loss of microRNAs in neural crest leads to cardiovascular syndromes resembling human congenital heart defects. Arterioscler Thromb Vasc Biol 30: 2575-2586, 2010.

57. Tu Y, Wan L, Bu L, et al: MicroRNA-22 downregulation by atorvastatin in a mouse model of heart hypertrophy: a new mechanism for antihypertrophic intervention. Cell Physiol Biochem 31: 997-1008, 2013.

58. Stankunas K, Ma GK, Kuhnert FJ, Kuo CJ and Chang CP: VEGF signaling has distinct spatiotemporal roles during heart valve development. Dev Biol 347: 325-336, 2010.

59. Sheehy NT, Cordes KR, White MP, Ivey KN and Srivastava D The neural crest-enriched microRNA miR-452 regulates epithelial-mesenchymal signaling in the first pharyngeal arch. Development 137: 4307-4316, 2010.

60. Nagalingam RS, Sundaresan NR, Gupta MP, Geenen DL, Solaro RJ and Gupta M: A heart-enriched microRNA, miR-378, blocks heart hypertrophy by targeting Ras signaling. J Biol Chem 288: 11216-11232, 2013.

61. Boon RA, Iekushi K, Lechner S, et al: MicroRNA-34a regulates heart ageing and function. Nature 495: 107-110, 2013.

62. Li J, Cao Y, Ma X-j, et al: Roles of miR-1-1 and miR-181c in ventricular septal defects. Int J Cardiol 168: 1441-1446, 2013.

63. Xiao J, Liang D, Zhang H, et al: MicroRNA-204 is required for differentiation of human-derived cardiomyocyte progenitor cells. J Mol Cell Cardiol 53: 751-759, 2012.

64. Townley-Tilson W, Callis TE and Wang D: MicroRNAs 1, 133 , and 206: critical factors of skeletal and heart muscle development, function, and disease. Int J Biochem Cell Biol 42: 1252-1255, 2010.

65. Oliveira-Carvalho V, Carvalho VO and Bocchi EA: The emerging role of miR-208a in the heart. DNA Cell Biol 32: 8-12, 2013. 